\begin{tabular}{|c|l|}
\hline Title & Decoherence of exciton complexes in single InA IA s quantum dots measured by Fourier spectroscopy \\
\hline Author(s) & A dachi, S.; Y atsu, N.; Kaji, R.; Muto, S.; Sasakura, H. \\
\hline Citation & $\begin{array}{l}\text { A pplied Physics Letters, 91(16), 161910 } \\
\text { https://doi.org/10.1063/2800373 }\end{array}$ \\
\hline Issue Date & $2007-10$-15 \\
\hline Doc URL & http://hdl.handle.net/2115/30263 \\
\hline Rights & $\begin{array}{l}\text { Copyright 2007 A merican Institute of Physics. This article may be downloaded for personal use only. A ny other use } \\
\text { requires prior permission of the author and the A merican Institute of Physics. }\end{array}$ \\
\hline Type & article \\
\hline File Information & APL91-16.pdf \\
\hline
\end{tabular}

Instructions for use 


\title{
Decoherence of exciton complexes in single InAIAs quantum dots measured by Fourier spectroscopy
}

\author{
S. Adachi, ${ }^{a)}$ N. Yatsu, R. Kaji, and S. Muto ${ }^{\text {b) }}$ \\ Department of Applied Physics, Hokkaido University, Sapporo 060-8628, Japan \\ H. Sasakura \\ CREST, Japan Science and Technology Agency, Kawaguchi 332-0012, Japan
}

(Received 28 August 2007; accepted 28 September 2007; published online 17 October 2007)

\begin{abstract}
We report the single-photon Fourier spectroscopy of exciton and exciton complexes in single self-assembled InAlAs/AlGaAs quantum dots. A Michelson interferometer was inserted in the photoluminescence path for measuring the coherence length of the time-averaged emission of neutral excitons and their complexes. The effects of exchange interactions and fluctuations in the surrounding excess charges were estimated by comparing the coherence times and the excitation energies of the excitons and their complexes. (C) 2007 American Institute of Physics.
\end{abstract}

[DOI: $10.1063 / 1.2800373$ ]

Single quantum dot (QD) devices attract considerable attention in the field of semiconductor nanostructures because of their remarkable potential for single-photon emission, single-electron storage, and manipulation of single qubits for quantum information processing, which rely on discrete energy level of the QD due to its three-dimensional (3D) quantum confinement. ${ }^{1}$ However, a semiconductor QD can interact with its environment through carrier-phonon, carrier-carrier, and electron-nuclei interactions. In particular, electron-hole exchange interaction and electron-nuclei interactions are supposed to be enhanced due to the nanoscale 3D confinement. The suppression or positive utilization of the above interactions to realize an ideal isolated artificial atom is still a major challenge in the field of the semiconductor nanotechnology. The coherence time which is deduced from the coherence length or linewidth is an important parameter for the characterization of the single-photon wavepacket and emission and, therefore, to provide single-photon sources for quantum information processing. Currently, single-photon sources are an active research area, and the production of single photons on demand has been demonstrated using a variety of QDs with and without cavities in the visible to telecommunications wavelength range. ${ }^{2-8}$ Apart from such applications, the measurement of the coherence time provides fundamental physical information on excitons and exciton complexes with regard to the abovementioned interactions in QDs. In this paper, we report the effects of exchange interactions and fluctuation in the surrounding excess charges by comparing with the coherence times of exciton complexes in single self-assembled InAlAs QDs measured by the single-photon Fourier spectroscopy. The measurements of the coherence times of the positive trion and exciton complexes with an excited hole in a single QD have not been reported so far, and the presented data indicate clearly the effects of the exchange interactions on their coherence times.

The samples contain layers of $\operatorname{In}_{0.75} \mathrm{Al}_{0.25} \mathrm{As}$ QDs embedded in an $\mathrm{Al}_{0.3} \mathrm{Ga}_{0.7} \mathrm{As}$ barrier layer grown on a (100)

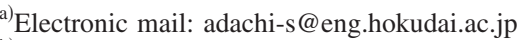

${ }^{b)}$ Also at CREST, Japan Science and Technology Agency, Kawaguchi 332-0012, Japan.
}

GaAs substrate by molecular beam epitaxy. The QDs are formed in the Stranski-Krastanow growth mode. For single-QD spectroscopy, small mesa structures with a typical top lateral size of $\sim 150 \mathrm{~nm}$ are fabricated. A cw-Ti:sapphire laser beam traveling along the QD growth direction is focused on the sample surface by a microscope objective. A Michelson interferometer inserted in the photoluminescence (PL) path is used to measure the coherence length of the time-averaged emission; this is a type of time-domain spectroscopy called single-photon Fourier spectroscopy and was first demonstrated by Kammerer et al. ${ }^{9}$ The obtained data show the variation of the interference fringe contrast (visibility) with the path-length difference between the two arms of the interferometer and give the magnitude of the Fourier transform of the intensity spectra. Fourier spectroscopy is an interesting method to explore the spectral dynamics of a single transition by both high temporal and high spectral resolutions with very low photon losses. ${ }^{9-12}$ The PL passing through the interferometer was dispersed by a triple-grating spectrometer $(f=0.64 \mathrm{~m})$ and was detected by a liquidnitrogen-cooled Si-charge-coupled device camera with a typical accumulation time of $500 \mathrm{~ms}$.

Figure 1(a) shows the contour plot of the PL intensity as a function of linear polarization angle at $0 \mathrm{~T}$; the PL intensity was obtained by rotating a half-wave plate before a fixed polarizer in front of the spectrometer. The sample was excited at the wetting layer (WL) of $\sim 1.698 \mathrm{eV}$ by the depolarized light. The figure shows almost all the spectral lines from a single InAlAs QD target for WL-excitation condition. Various measurements, including those of the quantities depicted in Fig. 1, indicate that the PL lines in the figure originate from the same single QD, and the PL lines are attributed to $X_{T}^{+}$(spin-triplet positive trion), $X X^{0}$ (neutral biexciton), $X X^{+}$(positively charged biexciton), $X^{0}$ (neutral exciton), and $X^{+}$(spin-singlet positive trion) from the low-energy side. ${ }^{13}$ Each line of the $X^{0}$ and $X X^{0}$ PLs has a clear fine structure and the dependence of linear polarization is anticorrelated; the high(low)-energy line of $X^{0}$ and the low(high)-energy line of $X X^{0}$ have the same $x(y)$ polarization. While the $X X^{0}$ state consists of spin-paired electrons and holes and is free from exchange interactions, the neutral exciton state splits due to anisotropic exchange interactions (AEI) resulting from the 

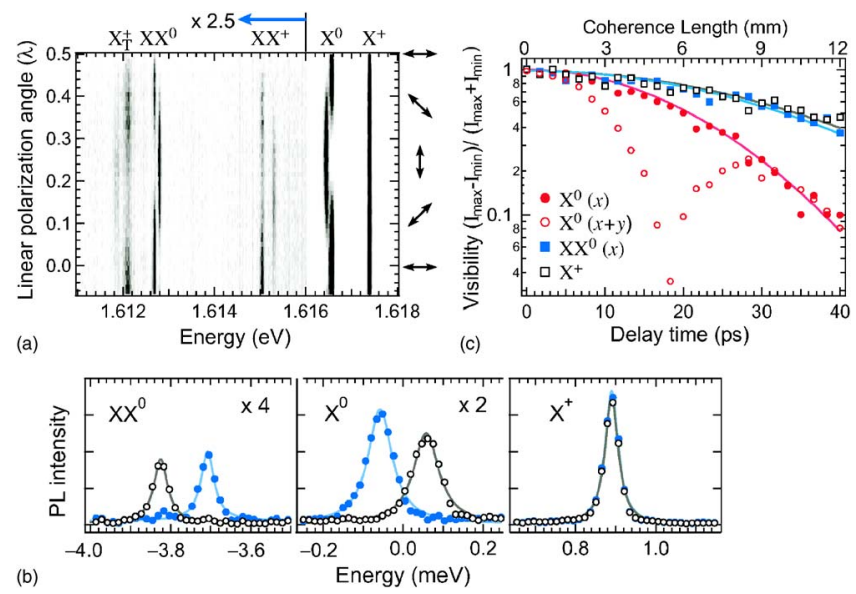

FIG. 1. (Color online) (a) PL intensity contour plot as a function of the linear polarization angle. The data were collected by the depolarized excitation at the WL excitation $(5 \mathrm{~K}, 0 \mathrm{~T})$. The PL polarization is depicted to the right of the figure. (b) PL spectra of the three main peaks $X X^{0}, X^{0}$, and $X^{+}$at a linear polarization angle of 0 and $\lambda / 4$, which are $x$ (open circles)- and $y$ (solid circles)-polarized PL components, respectively. Note that the energy axis is replotted from the midpoint of the two peaks of $X^{0}$. Clear fine structures are observed in the $X^{0}$ and $X X^{0}$ spectra, and no fine structure is observed for $X^{+}$. The linewidths (full width at half maximum) are 40,75, and $40 \mu \mathrm{eV}$ for $X X^{0}, X^{0}$, and $X^{+}$, respectively. (c) Typical visibility plot of the PLs of $X^{0}, X X^{0}$, and $X^{+}$at WL excitation. $x$ or $y$ in the parentheses indicates the polarization of the PL component. The solid lines are the fitting curves.

in-plane anisotropy of the overlap of the electron-hole wavefunctions; therefore, the PL spectral lines due to the transition from $X X^{0}$ to $X^{0}$ have the same splitting (but inverse polarization) as that for the transition from $X^{0}$ to the vacuum state. From the fitting in Fig. 1(b), the bright exciton splitting $\delta_{b}$ is found to be $115 \pm 5 \mu \mathrm{eV}$ and the binding energy of $X X^{0}$ is $+3.77 \mathrm{meV}$. $X^{+}$consists of two holes with the paired spin and one electron; therefore, exchange interaction is ineffective and $X^{+}$has no fine structure. Hence, $X^{+}$has a linewidth that is narrower than that of $X^{0}$, which suggests a long coherence of the electron spin. $X^{+}$has a negative binding energy $(-0.89 \mathrm{meV})$, which implies that a repulsive force acts between an exciton and a hole of $X^{+}$. The $X X^{+}$and $X_{T}^{+}$PLs are weak as compared to the others in this QD but show similar clear anticorrelated patterns. Here, $X X^{+}$PL implies the transition from $X X^{+}\left(J_{z}= \pm 3 / 2\right)$ to $X_{T}^{+}\left(J_{z}= \pm 1 / 2, \pm 5 / 2\right)$. Moreover, $X_{T}^{+} \quad \mathrm{PL}$ arises from the transition from $X_{T}^{+}\left(J_{z}= \pm 1 / 2, \pm 5 / 2\right)$ to the hole state $h\left(J_{z}= \pm 3 / 2\right) . X X^{+}$and $X_{T}^{+}$contain excited holes that occupy the $p$ shell, and exchange interactions between the $s$-shell and the $p$-shell carriers cause the state mixing and splitting, leading to a fine structure. $^{14,15}$

Figure 1(c) shows a typical visibility plot of the single-QD PL at the WL excitation. By varying the temporal delay $\tau$, the detected signal is given by $I(\tau)$ $=I_{0}\left\{1+V(\tau) \cos \left[E_{0} \tau / \hbar+\phi(\tau)\right]\right\}$, where $I_{0}$ is the average signal intensity, $E_{0}$ the central detection energy, $V(\tau)$ the interference fringe contrast (visibility) given by the modulus of the Fourier transform of the intensity spectrum, and $\phi(\tau)$ a slowly varying phase factor related to the line profile asymmetry. (An example of the rapid oscillation of the interference fringe is seen in the raw data in the upper panel of Fig. 3) In Fig. 1(c), $X^{0}(x)$ represented the $x$-polarized component and $X^{0}(x+y)$ represents both the $x$ - and $y$-polarized components. Since the InAlAs QD target has a relatively large bright exciton splitting $\delta_{b}$, the visibility of the whole Downloaded 16 Dec 2007 to 133.87.26.100. Redistribution subject
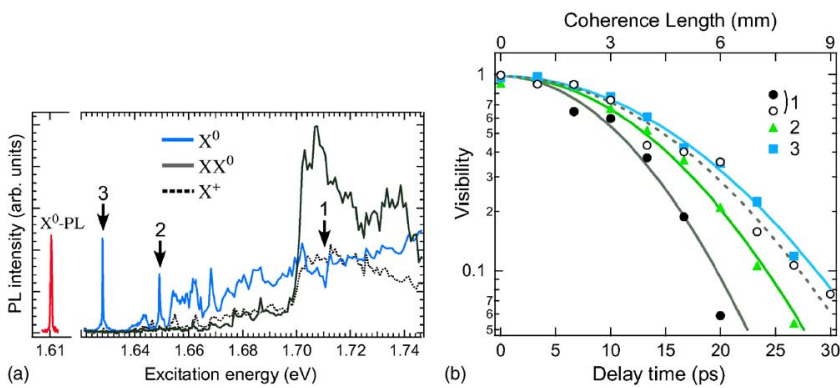

FIG. 2. (Color online) (a) PL excitation spectra at the detection energy of $X^{0}$. (b) Visibility plots of the PLs of the neutral exciton $X^{0}$ at different excitation energies: WL (solid circles) and 1 LO-resonant (triangles and squares) excitations. The respective excitation energies correspond to 1, 2, and 3 in (a). Only the $x$-polarized component was detected. The solid lines are the fitting curves.

fine structure components of $X^{0}$ and $X X^{0}$ oscillates with a period of $h / \delta_{b} \sim 36$ ps due to classical polarization interference. The visibility decay of $X^{0}[x($ or $y)]$ shows a clear Gaussian shape of $V(\tau)$ with a coherence time of $\sim 25 \mathrm{ps,}$ which indicates spectral fluctuations in the ground state energy. This is supposed to be due to the environmental charge because the effect reduces experimentally due to a near-resonant excitation such as the 1 LO resonant excitation discussed later (Fig. 2). Although the oscillatory and nonoscillatory behaviors of $X X^{0}$ are the same as those of $X^{0}$, depending on the detected polarization components, the coherence time of $X X^{0}$ is clearly different from that of $X^{0}$ and is longer $(\sim 40 \mathrm{ps})$, which is consistent with the highresolution PL spectra. In the case of $X^{+}$, the coherence time is long and is almost the same as that of $X X^{0}[x($ or $y)]$. The long coherence times of $X^{+}$and $X X^{0}$ as compared with $X^{0}$ are probably due to different exchange interactions between the electron and the hole in an exciton since $X X^{0}$ and $X^{+}$contain the paired holes with antiparallel spins; therefore, the exchange effect is ineffective. Since the coherence time $T_{2}$ is generally represented as $1 / T_{2}=1 /\left(2 T_{1}\right)+1 / T_{2}^{\text {phonon }}+1 / T_{2}^{\text {fluct }}$ $+1 / T_{2}^{\text {exchange }}$, under a particular excitation condition, it is reasonable to assume that the spectral fluctuation effect $1 / T_{2}^{\text {fluct }}$ and phonon scattering $1 / T_{2}^{\text {phonon }}$ affect all the species equally. Furthermore, the lifetime $T_{1}$ is estimated to be $\sim 1 \mathrm{~ns}$ from the single-QD-PL measurements using the streak camera. ${ }^{13}$ Therefore, on this timescale, the difference of the coherence times between $X^{+}$(or $X X^{0}$ ) and $X^{0}$ corresponds to the electron-hole exchange effect $1 / T_{2}^{\text {exchange }}$, and $T_{2}^{\text {exchange }}$ is estimated to be $\sim 65$ ps. For other QDs in the same and different mesas in the same sample, almost similar results are obtained and the value of $T_{2}^{\text {exchange }}$ is in the range of 50-100 ps. As compared with quantum wells where $T_{2}^{\text {exchange }} \leqslant 5 \mathrm{ps}$, ${ }^{16}$ this smaller contribution of the exchange interaction in QDs indicates a low scattering rate due to the $3 \mathrm{D}$ confinement.

The change on the excitation energy from the WL to the interior of the QD allows us to observe the effect of the charge fluctuations around a QD. Figure 2(a) shows the PL excitation spectra of another InAlAs QD in the same sample at a detection energy set at the $X^{0}$ peak. Three excitation energies, denoted as 1, 2, and 3 in the figure, were selected; these energies correspond to the edge of WL, 1 LO (GaAs), and 1 LO (InAlAs) resonances, respectively. The respective visibility plots are shown in Fig. 2(b), where the case with a low-power excitation at the excitation energy of 1 is also plotted (open circles). In this QD, the coherence times are to AIP license or copyright; see http://apl.aip.org/apl/copyright.jsp 


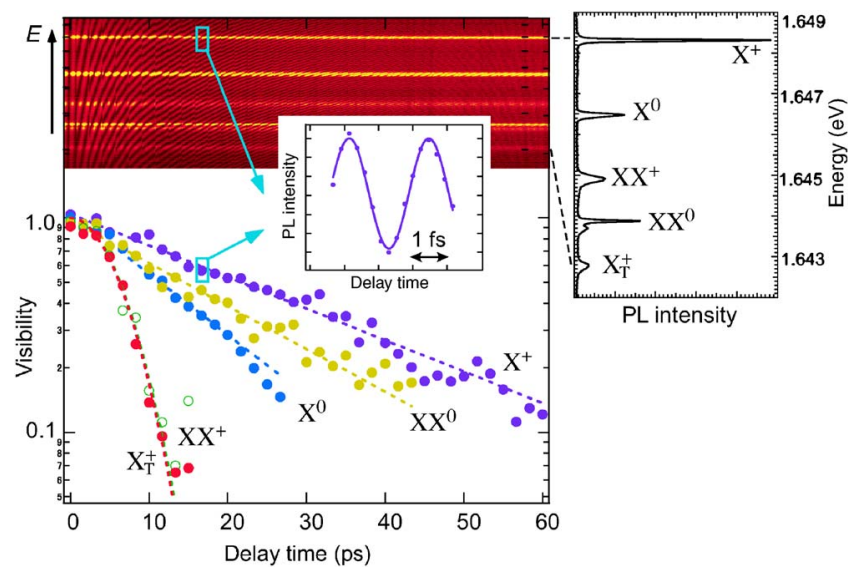

FIG. 3. (Color online) Visibility plot of the PLs of exciton and exciton complexes from the same single QD at WL excitation $(5 \mathrm{~K})$. The dashed lines are the fitting curves. The raw interferogram (upper panel) and the time-integrated spectra (right panel) are also shown.

19,16 , and 13 (18) ps at the excitation energies of 3,2, and 1 (the case at one-fifth of the excitation power), respectively. As observed in the figure, the visibility decays with the Gaussian profile in all the cases, but the coherence time increases with a decrease in the excitation energy. In addition, the coherence time depends on the excitation power. Since the optically excited carrier density decreases according to the density of states with a decrease in the excitation energy and since the local environment of the QD target changes, the above observations are supposed to be due to the effect of environmental charge fluctuation. Although it is difficult to provide the quantitative explanation for the effect of the charge fluctuation owing to difference in the local environment from one QD to another even in the same sample, the effect is observed in almost all the observed QDs; therefore, further research is necessary.

Finally, we verified the effect of AEI for $X X^{+}$and $X_{T}^{+}$. Figure 3 shows the visibility plot of another InAlAs QD in a different sample where the $X X^{+}$and $X_{T}^{+}$peaks can be detected by the interferometer even at a low excitation power. Actually, $X^{+}, X X^{0}$, and $X^{0}$ decay single exponentially in this QD. For this excitation condition, the effect of charge fluctuation appears to be reduced to the maximum extent possible. The coherence times are in the order of $X^{+}(35 \mathrm{ps})>X X^{0}$ $(25 \mathrm{ps})>X^{0}(17 \mathrm{ps})>X X^{+} \sim X_{T}^{+}(8 \mathrm{ps})$ for this QD. As mentioned above, $X X^{+}$and $X_{T}^{+}$contain excited holes; therefore, the AEI is applicable. ${ }^{14,15}$ The data indicate that the AEI induced by the $p$ shell is strong and results in a short coherence. The anisotropic exchange energies is $30 \pm 5$ and $62 \pm 5 \mu \mathrm{eV}$ for $X^{0}$ and $X_{T}^{+}$, respectively, for this QD; these energies are obtained by detailed spectral measurements under zero and nonzero magnetic fields and from a similar analysis of $X X^{-}$by Akimov et al. ${ }^{15}$ Additionally, for the mag- netic fields up to $3.5 \mathrm{~T}$, the coherence time of $X^{+}$did not change for circular and linear excitations $(\sim 43 \mathrm{ps})$, which suggests that the coherence of $X^{+}$is not decided by the electron-nuclear spin interaction; thus, the only possible origin is phonon scattering.

We report the single-photon Fourier spectroscopy of excitons and exciton complexes in single self-assembled InAlAs/AlGaAs quantum dots. The measurement of the coherence times of the positive trion and exciton complexes with an excited hole was demonstrated for the first time. A longer coherence time is exhibited by $X X^{0}$ and $X^{+}$as compared with the others, and this strongly suggests that decoherence by the AEI of $s$ and $p$ shells is predominant in $X^{0}$, $X X^{+}$, and $X_{T}^{+}$; this predominance of decoherence is due to the fact that $X X^{0}$ and $X^{+}$have spin-paired holes, which ensures that the exchange interaction is effective. All the presented data suggest that the order of decoherence in the single InAlAs QD can be understood by the exchange effect. From their excitation energy dependence and Gaussian shaped visibility curve, the effect of fluctuations in the environmental excess charges is found to be important for this type of measurements.

\footnotetext{
${ }^{1}$ Semiconductor Spintronics and Quantum Computation, edited by D. D. Awschalom, N. Samarth, and D. Loss (Springer, Berlin, 2002).

${ }^{2}$ P. Michler, A. Kiraz, C. Becher, W. V. Schoenfeld, P. M. Petroff, L. Zhang, E. Hu, and A. Imamoglu, Science 290, 2282 (2000).

${ }^{3}$ C. Santori, M. Pelton, G. Solomon, Y. Dale, and Y. Yamamoto, Phys. Rev. Lett. 86, 1502 (2001).

${ }^{4}$ V. Zwiller, H. Blom, P. Jonsson, N. Panev, S. Jeppesen, T. Tsegaye, E. Goobar, M.-E. Pistol, L. Samuelson, and G. Björk, Appl. Phys. Lett. 78, 2476 (2001).

${ }^{5}$ Z. Yuan, B. E. Kardyanal, R. M. Stevenson, A. J. Shields, C. J. Lobo, K. Cooper, N. S. Beattie, D. A. Ritchie, and M. Pepper, Science 295, 102 (2002).

${ }^{6}$ V. Zwiller, T. Aichele, W. Seifert, J. Persson, and O. Benson, Appl. Phys. Lett. 82, 1509 (2003).

${ }^{7}$ K. Takemoto, Y. Sakuma, S. Hirose, T. Usuki, N. Yokoyama, T. Miyazawa, M. Takatsu, and Y. Arakawa, Jpn. J. Appl. Phys., Part 2 43, L993 (2004)

${ }^{8}$ S. Kimura, H. Kumano, M. Endo, I. Suemune, T. Yokoi, H. Sasakura, S. Adachi, S. Muto, H. Z. Song, S. Hirose, and T. Usuki, Jpn. J. Appl. Phys., Part 2 44, L793 (2005).

${ }^{9}$ C. Kammerer, G. Cassabois, C. Voisin, M. Perrin, C. Delalande, Ph. Roussignol, and J. M. Gérard, Appl. Phys. Lett. 81, 2737 (2002).

${ }^{10}$ C. Santori, D. Fattal, J. Vučković, G. S. Solomon, and Y. Yamamoto, Nature (London) 419, 594 (2002).

${ }^{11}$ V. Zwiller, T. Aichele, and O. Benson, Phys. Rev. B 69, 165307 (2004).

${ }^{12}$ K. Kuroda, T. Kuroda, K. Sakoda, K. Watanabe, N. Koguchi, and G. Kido, Appl. Phys. Lett. 88, 124101 (2006).

${ }^{13}$ H. Kumano, S. Kimura, M. Endo, H. Sasakura, S. Adachi, S. Muto, and I. Suemune, J. Nanoelectron. Optoelectron. 1, 39 (2006).

${ }^{14}$ K. V. Kavokin, Phys. Status Solidi A 95, 592 (2003).

${ }^{15}$ I. A. Akimov, K. V. Kavokin, A. Hundt, and F. Henneberger, Phys. Rev. B 71, 075326 (2005).

${ }^{16}$ S. Adachi, T. Miyashita, S. Takeyama, Y. Takagi, and A. Tackeuchi,
} J. Lumin. 72/74, 307 (1997). 